\title{
Variabilidad iónica y características tróficas del embalse de Abreus, Cuba
}

\author{
Carmen Betancourt ${ }^{1, *}$, Roberto Suárez ${ }^{2}$ y Liliana Toledo ${ }^{1}$ \\ ${ }^{1}$ Centro de Estudios Ambientales de Cienfuegos. Calle 17, esq. Ave 46 s/n, Reparto Reina. Cienfuegos 55100, \\ Cuba. \\ ${ }^{2}$ Universidad de Cienfuegos. Carretera a Rodas km3. C.P. 59430 Cienfuegos, Cuba. \\ * Autor responsable de la correspondencia: carmen@gestion.ceac.cu
}

Recibido: 22/5/09

Aceptado: $10 / 6 / 10$

\begin{abstract}
Ionic variability and trophic characteristics of the Abreus reservoir, Cuba

The Abreus reservoir is located in the Cienfuegos province, in mid-southern Cuba. It was built in 1986 for irrigation purposes but at present it is also used for industrial purposes. It is affected by agricultural, human and industrial wastes. In order to investigate the ionic variation, a temporal series of main compounds taken from a database generated from 1987 to 2005 was used. The descriptive statistics, seasonal behavior, and the relationship among variables were evaluated, and a principal component analysis was performed for data reduction. Water was slightly saline and very hard, with a high correlation among variables, which allowed them to be grouped in a new variable called "mineralization". This new variable had a marked seasonal behavior with significant differences between two periods, from November to May, in which the water has a higher saline content and is influenced by evaporation, and from June to October, when the composition of the water is influenced by the rain. The values obtained for the seasonal indexes of the variables studied corroborate the significant differences in the mineralization between both periods. This information is considered relevant for better water management. To evaluate the trophic status, five sampling campaigns were carried out between 2008 and 2009, in which water transparency was measured using Secchi's Disc, as well as total phosphorous $\left(\mathrm{P}_{T}\right)$ concentration, total inorganic nitrogen $\left(\mathrm{Ni}_{T}\right)$, and chlorophyll $a$. In spite of the high values of hardness, $\mathrm{P}_{T}$ registered high values, classifying the reservoir as eutrophic. The nitrogen was the limiting nutrient for the phytoplanktonic growing. For this reason it must be regulated in order to control the reservoirs eutrophication.
\end{abstract}

Key words: Major ions, seasonal behavior, seasonal indexes, trophic state, Abreus reservoir.

\section{RESUMEN}

\section{Variabilidad iónica y características tróficas del embalse Abreus, Cuba}

El embalse Abreus está ubicado en la provincia de Cienfuegos, al centro-sur de Cuba. Fue construido en 1986 con el objetivo de utilizar sus aguas para el riego, pero actualmente tiene también uso industrial. Está afectado por residuales de origen agropecuario, humano e industrial. Para investigar la variabilidad iónica se utilizó una serie temporal de compuestos mayoritarios partiendo de datos generados entre los años 1987 y 2005, se evaluaron los estadísticos descriptivos, el comportamiento estacional, las relaciones entre variables y se realizó un análisis de componentes principales para la reducción de variables. El agua resultó medianamente salina y de dureza elevada, con una alta correlación entre las variables, lo que permitió su agrupación en una nueva variable denominada mineralización, con marcado comportamiento estacional, diferenciando claramente dos períodos a lo largo del año, de Noviembre a Mayo, en la que el agua tiene una mayor concentración de sales y está influenciada por la evaporación, y de Junio a Octubre, cuando la composición del agua está influenciada por la lluvia. Los valores obtenidos para los índices estacionales de las variables estudiadas, corroboran las diferencias significativas en la mineralización para los dos períodos ya mencionados. Esta información se considera que es relevante para realizar una mejor gestión del agua. Para evaluar el estado trófico se realizaron cinco muestreos entre los años 2008 y 2009 en los que se midió la transparencia del agua mediante el disco de Secchi, así como las concentraciones de fósforo total $\left(P_{T}\right)$, nitrógeno total inorgánico $\left(\mathrm{Ni}_{T}\right)$ y clorofila a. A pesar de los altos valores de dureza del agua, el $P_{T}$ registró valores elevados, clasificándose al embalse como eutrófico. El nitrógeno fue el nutriente que limitó el desarrollo fitoplanctónico por lo cual debe ser regulado para controlar la eutrofia del embalse.

Palabras clave: Iones mayoritarios, comportamiento estacional, índices estacionales, estado trófico, embalse Abreus. 


\section{INTRODUCCIÓN}

La calidad del agua está determinada por sus propiedades físicas, químicas y biológicas cuantificadas por diferentes variables medibles o analizables. Igualmente hay que considerar que estas propiedades están afectadas por factores externos como pueden ser la topografía, el clima (Gibbs, 1970) y, de forma especial, la actividad humana en la cuenca.

La calidad del agua está determinada por sus propiedades físicas, químicas y biológicas cuantificadas por diferentes variables medibles o analizables. Igualmente hay que considerar que estas propiedades están afectadas por factores externos como pueden ser la topografía, el clima (Gibbs, 1970) $y$, de forma especial, la actividad humana en la cuenca. Finalmente es importante considerar que la calidad del agua está estrechamente relacionada con el tipo de uso que se le quiere dar.

Para hacer una adecuada determinación de la calidad del agua y establecer un manejo eficiente del recurso, debe conocerse el comportamiento estacional de las variables físicas, químicas y biológicas, ya que a lo largo del año registran una gran variabilidad que debe ser medida.

Cuando el comportamiento estacional es importante se producen diferencias significativas en las características fisico-químicas del agua, que pueden causar inconvenientes para su uso y tratamiento. El conocimiento de la variabilidad de los índices estacionales de los componentes mayoritarios del agua es un elemento de gran importancia para su gestión.

Existen diversas herramientas estadísticas para evaluar estacionalidad (Liou et al., 2003; Wei, 2005; Shumway \& Dtoffer, 2006), en series de tiempo, que son usadas generalmente para planear y diseñar proyectos ambientales.

Diversos investigadores han referido la existencia de patrones estacionales en variables físico-químicas del agua (Dirnberger \& Weinberger, 2005; Balistrieri et al., 2006; Kim \& Kim, 2006; Serafim et al., 2006; Kim et al., 2006; Mwaura, 2006; Langlet et al., 2007; Tapia et al., 2008; Bouza et al., 2008; Atobatele \& Ugwumba, 2008) y biológicas (Medina \& Herrera, 2003; De León \& Chalar, 2003; Rakocevic \& Hollert,
2005; Copetti et al., 2006). Sin embargo no es frecuente la evaluación de su magnitud.

En este trabajo se pretende evaluar la estacionalidad de los componentes mayoritarios y determinar los índices estacionales a partir de una serie temporal de 19 años en el embalse Abreus, así como su estado trófico mediante mediciones realizadas durante un ciclo anual.

\section{MATERIAL Y MÉTODOS}

\section{Área de estudio}

El embalse Abreus fue construido en 1986 con el objetivo de utilizar sus aguas en el riego agrícola. En la actualidad está destinado además, para el abastecimiento de industrias localizadas en la cercanía de la ciudad de Cienfuegos.

Las dos corrientes de mayor aporte de agua al embalse son los ríos Damují y Jabacoa (Fig. 1) y recibe casi la totalidad del agua que escurre por la cuenca, al ubicarse en el último tramo del río principal (Damují). Su cuenca yace sobre formaciones geológicas compuestas por arcillas, margas y calizas principalmente, así como basaltos, rocas epiclásticas y areniscas en menor proporción (Laiz y Flores, 2007).

Comas et al. (2004) encontraron que las aguas del río Damují, son ricas en electrolitos (cloruros, sulfatos, bicarbonatos, calcio y magnesio), de probable origen geológico, y no por contaminación.

En la cuenca del embalse se generan residuales de origen industrial, agropecuario y humano que fueron parcialmente tratados y afectaron la calidad del agua del mismo. Laiz y Flores (2007) encontraron altas densidades de cianobacterias (algas verde-azules), altos valores de fósforo total $\left(\mathrm{P}_{T}\right)$ de $0.049 \mathrm{mg} / \mathrm{L}$, baja transparencia al Disco Secchi (DS) de $0.8 \mathrm{~m}$ y elevadas concentraciones de clorofila $a 23.6 \mu \mathrm{g} / \mathrm{L}$, clasificando al embalse como eutrófico según la metodología de la OECD (1982).

Algunas características del embalse y su cuenca aparecen en la Tabla 1 (información brindada por la Empresa de Aprovechamiento Hidráulico en la provincia de Cienfuegos). 


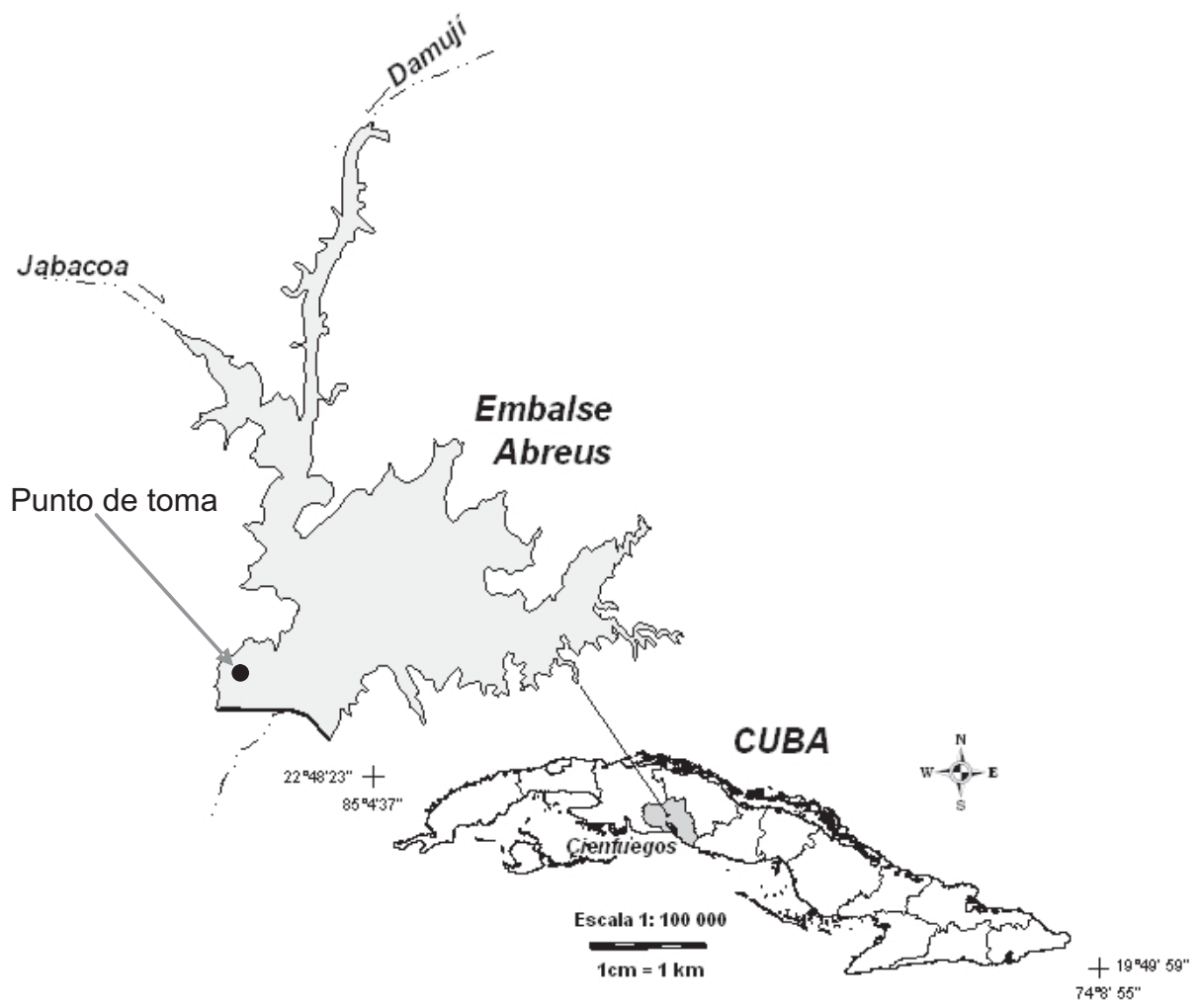

Figura 1. Área de estudio. Embalse Abreus, en el centro de la provincia Cienfuegos. Study site. Abreus reservoir in the centre of the Cienfuegos province.

\section{Muestreo y ensayos}

La base de datos de la serie temporal procede de la Red de Calidad de las Aguas de la Empresa de Aprovechamiento Hidráulico de Cien-

Tabla 1. Algunas características del área de estudio. Some characteristics of the study area.

\begin{tabular}{lc}
\hline \multicolumn{2}{c}{ Características del área de estudio } \\
\hline Área de la cuenca $\left(\mathrm{km}^{2}\right)$ & 1075 \\
Altitud media de la cuenca $(\mathrm{m})$ & 50 \\
Altitud del embalse $(\mathrm{m})$ & 10 \\
Volumen del embalse $\left(\mathrm{hm}^{3}\right)$ & 35 \\
Área del embalse $\left(\mathrm{km}^{2}\right)$ & 5.40 \\
Área de la cuenca/Área del embalse & 199 \\
Escurrimiento medio anual $\left(\mathrm{hm}^{3}\right)$ & 194.4 \\
Gastos del río máximo $\left(\mathrm{m}^{3} / \mathrm{seg}\right)$ & 124 \\
Longitud del río (km) & 52.8 \\
Profundidad máxima del embalse (m) & 12.5 \\
Nivel promedio de la superficie del embalse & \\
durante los muestreos (msnm) & 8.70 \\
Profundidad del punto de toma (msnm) & 7.79 \\
\hline
\end{tabular}

fuegos y corresponde con mediciones mensuales del agua procedente de la toma de agua en el embalse. Incluye, conductividad eléctrica $(\mathrm{CE})$, dureza total $\left(\mathrm{D}_{T}\right)$, los iones bicarbonato $\left(\mathrm{HCO}_{3}\right)$, cloruro $(\mathrm{Cl})$, calcio $(\mathrm{Ca})$, magnesio $(\mathrm{Mg})$, y sodio más potasio $(\mathrm{Na}+\mathrm{K})$ y se enmarca entre los años 1987-2006.

Para evaluar el estado trófico del embalse se tomaron muestras en la superficie de la columna de agua donde se localiza el punto de toma y a la profundidad del punto de toma (ubicado a $1 \mathrm{~m}$ de la superficie como promedio), durante los meses de Abril, Agosto, Octubre y Diciembre de 2008 y Marzo de 2009, para los ensayos de fósforo total $\left(\mathrm{P}_{T}\right)$, ortofosfatos $\left(\mathrm{P}-\mathrm{PO}_{4}\right)$, nitratos $\left(\mathrm{N}-\mathrm{NO}_{3}\right)$, nitrito $\left(\mathrm{N}-\mathrm{NO}_{2}\right)$, nitrógeno amoniacal $\left(\mathrm{N}-\mathrm{NH}_{3}\right)$ y clorofila $a$.

En la toma de muestras se utilizó una botella Niskin de cinco litros de capacidad; la transparencia del agua se midió con un disco de Secchi de $20 \mathrm{~cm}$ de diámetro. 
La concentración de $\mathrm{N}-\mathrm{NH}_{4}$ se determinó por formación de indofenol azul, el $\mathrm{N}-\mathrm{NO}_{2}$ por diazotación con sulfanilamida, el $\mathrm{N}-\mathrm{NO}_{3}$ por reducción con hidracina, el $\mathrm{P}-\mathrm{PO}_{4}$ se cuantificó por formación de un complejo azul con molibdato de amonio, el $\mathrm{P}_{T}$ por reducción con ácido ascórbico y la clorofila $a$ por fluorescencia.

Los ensayos se realizaron sin filtrar las muestras por lo que incluyen los nutrientes suspendidos y disueltos. Los límites de cuantificación (LC) expresados en $\mathrm{mg} / \mathrm{l}$, fueron $\mathrm{N}-\mathrm{NH}_{4}$; $0.045, \mathrm{~N}-\mathrm{NO}_{2} ; 0.001, \mathrm{~N}-\mathrm{NO}_{3} ; 0.006, \mathrm{P}-\mathrm{PO}_{4}$; $0.005, \mathrm{P}_{T} ; 0.013$. Los valores menores que el límite de cuantificación se consideraron cero para la confección de los gráficos.

Estos ensayos fueron realizados en el laboratorio del Centro de Estudios Ambientales de Cienfuegos según las especificaciones de APHA (1998).

\section{Tratamiento estadístico}

Para el tratamiento estadístico se empleó el Statistical Package for Social Sciences (SPSS) en su versión 15.0. Se calcularon los descriptivos mínimo, máximo, media y desviación típica. Se usó el coeficiente de correlación de Pearson para el análisis de correlaciones entre las variables; en el estudio de la estacionalidad de las series se empleó el recurso de los diagramas de secuencia, el correlograma y el periodograma. El cálculo de los índices estacionales se realizó considerando un modelo aditivo y estático y se aplicó el método de relación con la tendencia. Cada índice es un promedio de la diferencia entre el valor de la serie y la línea de tendencia calculada para cada mes. Por tanto representa una cantidad en unidades (para la CE en $\mu \mathrm{mhos} / \mathrm{cm}$ y para el resto de las variables en $\mathrm{mg} / \mathrm{L}$ ) por encima (signo positivo) o por debajo (signo negativo) de la línea de tendencia que en este caso se calculó mediante las medias móviles.

Con el objetivo de disminuir la dimensionalidad del problema a investigar se estudió el sistema de relaciones entre las variables de forma conjunta mediante un análisis factorial por el método de componentes principales y mediante rotación varimax.

Para verificar la existencia de diferencias significativas entre las variables en los períodos de
Junio-Octubre y Noviembre-Mayo y para comparar las concentraciones de $\mathrm{P}-\mathrm{PO}_{4}$ y $\mathrm{P}_{T}$ en la superficie y punto de toma del embalse se aplicó la prueba $t$ de Student para dos muestras independientes, con un nivel de significación de 0,05 y con previa verificación del supuesto de normalidad.

El valor medio del tiempo de residencia del agua en el embalse se calculó mediante la expresión que se obtuvo al dividir el volumen de agua almacenada por el flujo de salida diario en el período 2007-2009. Este procedimiento, es tradicional y fue empleado por otros autores (Foy, 1992; Sivadier et al., 1994; Straskraba et al., 1995; Betancourt et al., 2009).

\section{Procedimiento usado para la clasificación trófica de las aguas}

La clasificación trófica del embalse se realizó mediante un índice de estado trófico (IET) sugerido por Carlson (1977) y modificado por Toledo et al (1983) para zonas tropicales. Las ecuaciones usadas en el cálculo de estos índices para la transparencia (DS), $\mathrm{P}_{T}, \mathrm{PO}_{4}$ y clorofila $a$ (Clf $a$ ) y el índice de estado trófico medio que combina los índices de cada una de las variables mencionadas se expresan:

$$
\begin{aligned}
& I E T_{\text {Modificado }}(\mathrm{DS})=10 *\left[6-\left(\frac{0.64+\ln (D S)}{\ln 2}\right)\right] \\
& I E T_{\text {Modificado }}\left(\mathrm{P}_{T}\right)=10 *\left[6-\frac{\ln \left(\frac{80.32}{P_{T}}\right)}{\ln 2}\right] \\
& I E T_{\text {Modificado }}\left(\mathrm{PO}_{4}\right)=10 *\left[6-\left(\frac{\ln \left(\frac{21.67}{\mathrm{PO}_{4}}\right)}{\ln 2}\right)\right] \\
& I E T_{\text {Modificado }}(\mathrm{Cllf} a)= \\
& I 0 *\left[6-\left(\frac{2.04-0.695 \ln \mathrm{Clf} a}{2 a}\right)\right] \\
& I E T_{\text {Modificado }}(\text { medio })== \\
& \frac{I E T(\mathrm{DS})+2\left[\left(I E T P_{T}\right)+\operatorname{IET}\left(\mathrm{PO}_{4}\right)+\operatorname{IET}(\mathrm{Clf} a)\right]}{7}
\end{aligned}
$$


Tabla 2. Estadísticos descriptivos de los componentes mayoritarios del embalse Abreus. Descriptive statistics of major ions of the Abreus reservoir.

\begin{tabular}{ccccccccc}
\hline \multicolumn{7}{c}{ Estadísticos descriptivos de los componentes mayoritarios } \\
\hline & $\mathrm{CE}$ & $\mathrm{HCO}_{3}$ & $\mathrm{Cl}$ & $\mathrm{SO}_{4}$ & $\mathrm{Ca}$ & $\mathrm{Mg}$ & $(\mathrm{Na}+\mathrm{K})$ & $\mathrm{D}_{T}$ \\
\hline Máximo & 745 & 327 & 63 & 53 & 94 & 22 & 49 & 318 \\
Media & 554 & 235 & 41 & 33 & 64 & 13 & 35 & 216 \\
Mínimo & 380 & 152 & 10 & 19 & 32 & 6 & 16 & 130 \\
Desviación típica & 73 & 37 & 7 & 6 & 12 & 3 & 5 & 37 \\
$n$ & 233 & 233 & 233 & 233 & 233 & 233 & 233 & 233 \\
\hline
\end{tabular}

Con el resultado del cálculo del IET Modificado (medio) se clasificó el estado trófico de las aguas según el siguiente intervalo: Oligotrofia $\leq 44$; Mesotrofia $44<$ IET $<54$ y Eutrofia $\geq 54$.

\section{RESULTADOS}

\section{Evaluación de la serie temporal}

\section{Estadísticos descriptivos}

En la Tabla 2 se muestran los valores máximos, mínimos, medios y desviaciones típicas para los componentes mayoritarios. En todas las variables analizadas se observó alta dispersión de los valores. Según las medias de $\mathrm{CE}$ y de $\mathrm{D}_{T}$ el agua resultó medianamente salina de acuerdo con la clasificación de Serruya \& Pollingher (1983) y de dureza elevada según la clasificación de Durfor y Becker (1964), con predominio de los iones de $\mathrm{Ca}$ y $\mathrm{HCO}_{3}$.

\section{Correlación entre variables}

Las correlaciones entre variables fueron positivas y significativas en todos los casos $(p<0.01)$. Los valores más altos se encontraron entre la $\mathrm{CE}$ y el resto de las variables, excepto el $\mathrm{Mg}$ que fue el ión con menor correlación. Hubo una alta correlación entre el Ca y el $\mathrm{HCO}_{3}$ (Tabla 3).

\section{Estacionalidad}

Se registró estacionalidad en todas las variables estudiadas, según la significación estadística del coeficiente de autocorrelación de orden 12 del correlograma (Fig. 2) y el valor prominente en la frecuencia 0.08 , equivalente a un período de 12 meses mostrado en el periodograma de la serie (Fig. 3).

La figura $2 \mathrm{~A}$ representa los coeficientes de autocorrelación de la serie de la variable $\mathrm{D}_{T}$ respecto al tiempo de retardo en meses. La misma mostró una autocorrelación significativa y periódica que alcanza un máximo con signo negati-

Tabla 3. Matriz de correlación de Pearson para los componentes mayoritarios. Pearson's correlation matrix for the major ions.

\begin{tabular}{|c|c|c|c|c|c|c|c|c|}
\hline \multicolumn{9}{|c|}{$\begin{array}{c}\text { Matríz de correlación de Pearson para los componentes mayoritarios }(n=233) \\
\text { Todas las correlaciones fueron significativas }(p<0.01) \text { y positivas }\end{array}$} \\
\hline & $\mathrm{CE}$ & $\mathrm{HCO}_{3}$ & $\mathrm{Cl}$ & $\mathrm{SO}_{4}$ & $\mathrm{Ca}$ & $\mathrm{Mg}$ & $\mathrm{D}_{T}$ & $\mathrm{Na}+\mathrm{K}$ \\
\hline CE & 1 & & & & & & & \\
\hline $\mathrm{HCO}_{3}$ & 0.822 & 1 & & & & & & \\
\hline $\mathrm{Cl}$ & 0.652 & 0.553 & 1 & & & & & \\
\hline $\mathrm{SO}_{4}$ & 0.625 & 0.575 & 0.449 & 1 & & & & \\
\hline $\mathrm{Ca}$ & 0.759 & 0.826 & 0.505 & 0.621 & 1 & & & \\
\hline $\mathrm{Mg}$ & 0.517 & 0.466 & 0.342 & 0.376 & 0.449 & 1 & & \\
\hline $\mathrm{D}_{T}$ & 0.782 & 0.824 & 0.522 & 0.620 & 0.958 & 0.677 & 1 & \\
\hline $\mathrm{Na}+\mathrm{K}$ & 0.651 & 0.632 & 0.577 & 0.500 & 0.567 & 0.383 & 0.586 & 1 \\
\hline
\end{tabular}



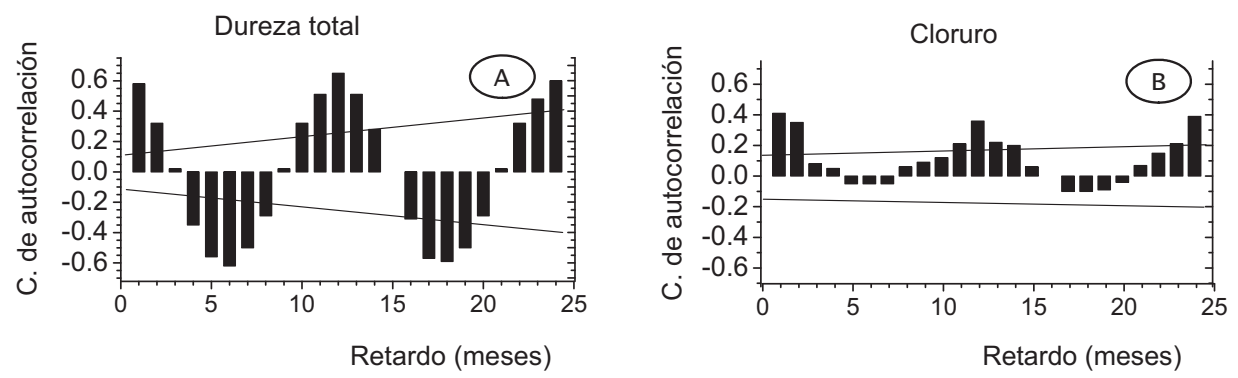

Figura 2. Autocorrelaciones estimadas para los componentes mayoritarios. A: Coeficiente de autocorrelación para la dureza total. Figuras similares se obtuvieron para el resto de las variables, incluyendo la mineralización y exceptuando los Cl. B: Coeficiente de autocorrelación para los cloruros. Estimated autocorrelations for the major components. A: Autocorrelation coefficient for total hardness. Similar figures were obtained for the rest of the variables including mineralization and excluding Cl. B: Autocorrelation coefficient estimated for Chlorides.

vo para un retardo de 6 y con signo positivo para un retardo de 12, esto significa que cada 6 meses la dureza total alterna los valores de máximos y mínimos y cada 12 meses los valores se correlacionan de forma positiva. Este patrón es similar para el resto de las variables, excepto los $\mathrm{Cl}$ que solo registraron un coeficiente de autocorrelación de orden 12 (Fig. 2B) lo cual significa que los valores se repitieron solo al transcurrir un año y no en los seis meses como en el resto de las variables.

Mediante la determinación de los índices estacionales se determinó la magnitud por exceso o por defecto de la media de los valores mensuales, respecto a la media general para cada una de las variables estudiadas.

El comportamiento de estos índices en los diferentes meses del año fue similar para la mayoría de las variables, los valores más altos se registraron en el mes de Enero, excepto $\mathrm{Cl}$,

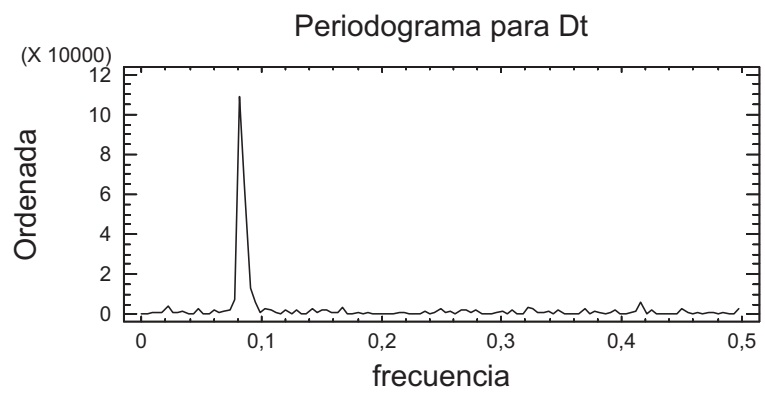

Figura 3. Periodograma para la dureza total. Figuras similares fueron obtenidas para el resto de las variables, incluyendo la mineralización. Periodogram for total hardness. Similar figures were obtained for the rest of variables, including mineralization.
$\mathrm{SO}_{4}$ y CE que registraron un máximo en Febrero, con una disminución gradual hasta Julio o Agosto, un aumento en Septiembre, con un registro mínimo en Octubre y un brusco aumento en los meses posteriores (Fig. 4).

Considerando la alta correlación observada entre todas las variables (Tabla 3), se aplicó un análisis factorial por el método de componentes principales. El índice de adecuacidad muestral para la realización del análisis factorial fue de 0.766 , con adecuaciones para cada variable superiores a 0.64 , exceptuando la variable $\mathrm{Mg}$ que mostró un índice de 0.46 .

En el análisis factorial la única componente con valor propio mayor que uno, explicó el $66.07 \%$ de la varianza total y fue nominado como el factor "mineralización de las aguas del embalse". Todas las variables mostraron altas correlaciones con la mineralización, lo cual permitió utilizar este componente.

Posteriormente se analizó la estacionalidad del factor "mineralización". Esta nueva variable mostró un comportamiento estacional bien definido similar al patrón seguido por los componentes mayoritarios representados en la figura $2 \mathrm{~A}$ y figura 3 .

Los índices estacionales para el factor "mineralización" se muestran en la figura 4 y siguieron el mismo patrón observado en las demás variables analizadas en la serie temporal.

La variabilidad de los índices estacionales de las variables estudiadas (incluyendo la "mineralización”), permitió diferenciar dos períodos de tiempo: Junio-Octubre con valores negativos de los índices y Noviembre-Mayo con los valores positivos 



Figura 4. Variación anual de los índices estacionales de los componentes mayoritarios y la mineralización. Annual variation of the seasonal indexes for the major components and the mineralization.

(Fig. 4). Para verificar estas diferencias entre ambos períodos se comparó el valor registrado por cada variable en cada período y también se encontró diferencia significativa $(p<0.05)$ entre períodos.

\section{Tiempo de residencia del agua en el embalse}

La ubicación del embalse al final de la cuenca del río Damují ocasionó que el tiempo de residencia
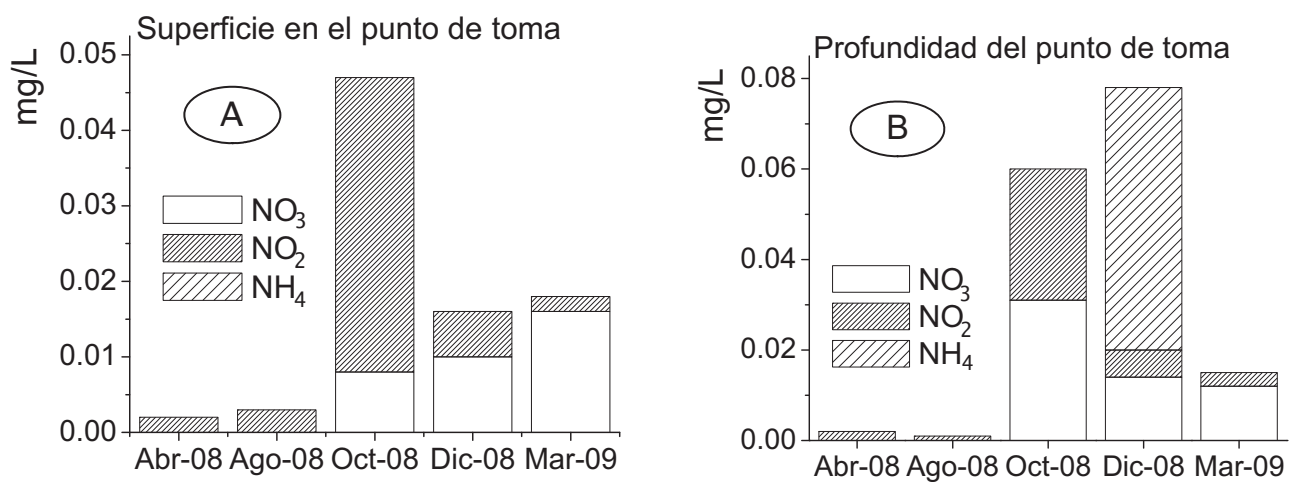

Figura 5. Composición del nitrógeno inorgánico en la columna de agua del punto de toma. A: Superficie. El N-NH $\mathrm{N}_{4}$ estuvo ausente en todos los muestreos. B: Punto de toma. El N- $\mathrm{NH}_{4}$ sólo fue registrado en el mes de Diciembre. Inorganic nitrogen composition in the water column of the outlet point. A: Surface. The $\mathrm{N}-\mathrm{NH}_{4}$ was absent in all samples. B: Outlet point. The $\mathrm{N}-\mathrm{NH} 4 \mathrm{was}$ only registered in December. 

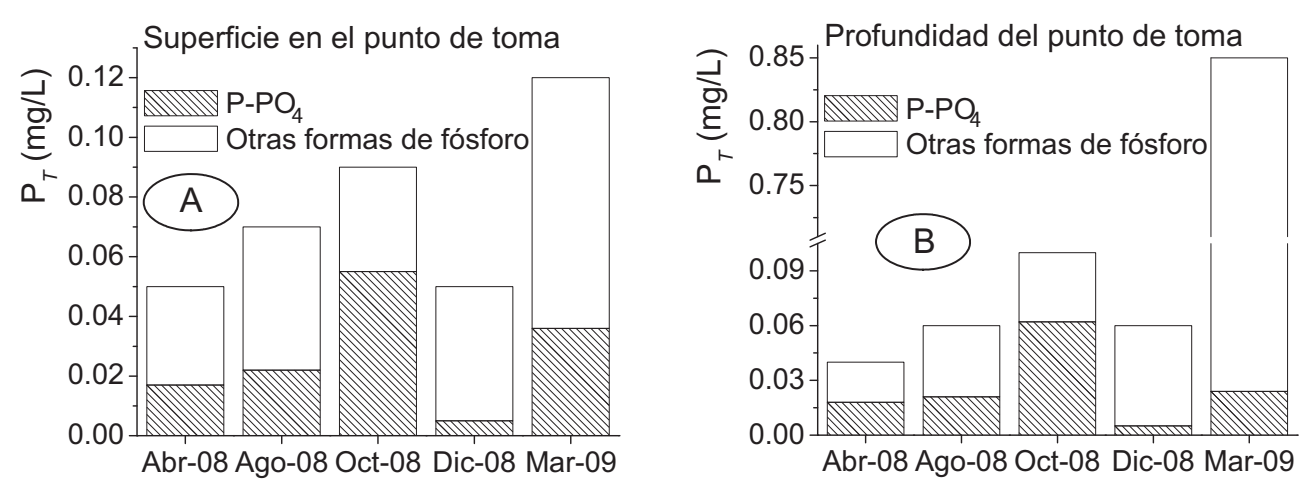

Figura 6. Proporción del $\mathrm{P}-\mathrm{PO}_{4}$ en relación al $\mathrm{P}_{T}$ en la columna de agua del punto de toma. A: Superficie. B: Punto de toma. Proportion of $\mathrm{P}_{-\mathrm{PO}_{4}}$ to $\mathrm{P}_{T}$ in the water column of the outlet point. A: Surface. B: Outlet point.

del agua en el embalse dependiera estrechamente de la pluviosidad en la cuenca de alimentación.

Según el procedimiento utilizado el agua permaneció en el embalse por 289 días como promedio durante la etapa que se estudió el estado trófico del embalse (2008-2009). El período comprendido entre Junio y Octubre (período lluvioso en Cuba) se caracterizó por una gran dispersión en los valores del tiempo de residencia; valores máximos equivalente a 1296 días con mínimo del orden de 0.43 días, para un valor medio de 213 días.

\section{Transparencia del agua y concentraciones de nitrógeno, fósforo y clorofila $a$}

La transparencia del agua osciló entre 0.5 y $1.85 \mathrm{~m}$. Las concentraciones de $\mathrm{P}_{T}, \mathrm{P}-\mathrm{PO}_{4}$, $\mathrm{N}-\mathrm{NO}_{3}, \mathrm{~N}-\mathrm{NO}_{2}$, y $\mathrm{N}-\mathrm{NH}_{3}$ no tuvieron diferencias significativas $(p>0.05)$ en ambas estaciones de muestreo (superficie y profundidad del punto de toma). El nitrógeno total inorgánico registró concentraciones comprendidas entre $0.002-0.047 \mathrm{mg} / \mathrm{l}$ en la superficie (Fig. 5A) y 0.001-0.079 en la profundidad del punto de toma (Fig. 5B). El N-NO $\mathrm{Nue}_{2}$ la única especie que siempre se detectó, mientras que el $\mathrm{N}-\mathrm{NH}_{4}$ sólo se cuantificó en la profundidad del punto de toma en el mes de Diciembre.

Las concentraciones de $\mathrm{P}_{T}$ fueron superiores a las de $\mathrm{Ni}_{T} ; 0.050-0.120 \mathrm{mg} / \mathrm{L}$ en la superficie del punto de toma (Fig. 6A) y 0.040-0.850 en la profundidad del punto de toma (Fig. 6B). El intervalo para el $\mathrm{P}-\mathrm{PO}_{4}$ fue $0.005-0.055 \mathrm{mg} / \mathrm{L}$ y 0.018-0.062 respectivamente. Se observó una tendencia al aumento de las concentraciones tanto para $\mathrm{P}_{T}$ como para el $\mathrm{P}-\mathrm{PO}_{4}$ con el transcurso del tiempo (Fig. 6A y 6B), exceptuando Diciembre cuando se registró una disminución de los valores. La fracción de $\mathrm{P}-\mathrm{PO}_{4}$ dentro del $\mathrm{P}_{T}$, siempre fue superior en los muestreos realizados en la superficie del punto de toma, respecto a las muestras tomadas en la profundidad del punto de toma. El mayor valor de la fracción se midió en el mes de Octubre y correspondió con el final del verano, en Diciembre (invierno en Cuba) se registró la menor proporción en ambas estaciones de muestreo.

Los valores de $\mathrm{P}-\mathrm{PO}_{4}$ no guardaron relación con la clorofila $a$. Esta última variable estuvo en un intervalo entre 31 y $42 \mu \mathrm{g} / \mathrm{L}$.

\section{Clasificación trófica y relación entre el $\mathrm{Ni}_{T}$ $\mathbf{y}$ el $\mathbf{P}_{T}$}

La aplicación del criterio de Toledo et al. (1983) para el cálculo del índice de estado trófico en este estudio reveló que de forma predominante las aguas embalsadas fueron eutróficas. El índice obtenido según la ecuación que incluye todas las variables fue de 64 (indicó eutrofia), para los valores individuales de $\mathrm{P}_{T}$, clorofila $a$ y $\mathrm{P}-\mathrm{PO}_{4}$ el valor de los índices clasificó al embalse como eutrófico (66, 58 y 75 respectivamente), para la transparencia fue de 51 (indicó mesotrofia).

En el acuatorio estudiado la relación $\mathrm{Ni}_{T}: \mathrm{P}_{T}$, obtenida a partir de las mediciones realizadas en la superficie estuvo en el intervalo 0.040-0.522. Estos resultados revelaron al nitrógeno como el nutriente que limitó el desarrollo fitoplanctónico. 


\section{DISCUSIÓN}

El predominio de los iones $\mathrm{Ca}$ y $\mathrm{HCO}_{3}$ así como la clasificación del agua como medianamente salina y de dureza elevada, está en correspondencia con la geología de la cuenca y los resultados obtenidos por Comas et al. (2004).

La diferencia significativa observada para la mineralización, entre los períodos Junio-Octubre y Noviembre-Mayo, debe ser considerada para un adecuado uso y manejo del recurso. Para usar esta agua en la industria o el riego agrícola hay que considerar las variaciones estacionales de los componentes mayoritarios. Por ejemplo la $\mathrm{D}_{T}$ en el mes de Enero fue superior al mes de Julio en $88.9 \mathrm{mg} / \mathrm{L}$ y los $\mathrm{Cl}$ con una media de $41 \mathrm{mg} / \mathrm{L}$, tuvo una diferencia entre los meses de Enero y Octubre de $15.3 \mathrm{mg} / \mathrm{L}$, valor que representó el $37.3 \%$ de su media. El diseño del tratamiento del agua y su gestión debe considerar estas diferencias, que fueron cuantificables mediante los índices estacionales de cada variable para cada mes.

La disminución de la mineralización del agua durante los meses comprendidos entre Junio y Octubre se relacionó con el período lluvioso en Cuba y con la ubicación geográfica del embalse (al final de la cuenca del río Damují).

Según Gibbs (1970), existen tres mecanismos naturales que explican la composición químicas de las aguas; la precipitación atmosférica, la solubilización de las rocas y la evaporación-precipitación, que pueden identificarse al graficar las SST en relación a la razón $\mathrm{Na} /(\mathrm{Na}+\mathrm{Ca})$ y $\mathrm{Cl} /\left(\mathrm{Cl}+\mathrm{HCO}_{3}\right)$.

Las aguas de este embalse de forma general y según los criterios de Gibbs (1970), se ubican en la zona que corresponde con la solubilización de las rocas. Para el período Noviembre-Mayo (temporada menos lluviosa), se observó una menor dispersión de los valores y un ligero acercamiento hacia la zona que corresponde con la evaporación-precipitación.

En Junio-Octubre se apreció un ligero acercamiento de las observaciones al área que corresponde con el predominio de la lluvia, según el modelo establecido por Gibbs (1970). Este es un período de abundantes precipitaciones en Cuba, pero también coincide con los registros de las temperaturas más altas, por lo que pueden veri- ficarse simultáneamente procesos de dilución de las sales disueltas (por la ocurrencia de las 1luvias) y procesos de concentración (por la evaporación que tiene lugar por las altas temperaturas). En este período predominó el proceso de dilución.

Los valores más bajos de la mineralización en el período Junio-Octubre podrían relacionarse también con la precipitación del $\mathrm{CaCO}_{3}$, debido a la alta demanda que se produce en este período por el fitoplancton, y/o por la disminución de su solubilidad al aumentar la temperatura del agua durante el verano. García et al. (2001), encontraron en un embalse mesotrófico de España, que la mayor sedimentación de $\mathrm{CaCO}_{3}$ ocurrió en el mes de Junio, coincidiendo con el verano.

La eutrofia encontrada en las aguas de este embalse se debe a la presencia en su cuenca de alimentación de fuentes de contaminación, con residuos ricos en nitrógeno y fósforo como la cría de ganado y asentamientos humanos.

Margalef (1982), señaló que aguas con concentraciones de Ca cercanas a $40 \mathrm{mg} / \mathrm{L}$ limitan fuertemente las concentraciones de $\mathrm{P}$ soluble, manteniéndolas en bajos niveles debido a su reactividad con los cationes. Además se ha propuesto que la precipitación del fosfato con el $\mathrm{CaCO}_{3}$ es el principal mecanismo que controla su solubilidad en aguas duras previniendo la eutrofización (Estrada, 1978).

Diversos estudios han confirmado la prevalencia del enlace del $\mathrm{Ca}$ con el ortofosfato en aguas procedentes de zonas cárstica (López \& Morgui, 1993; Chen \& Wu, 2007), convirtiéndose el P en el nutriente limitante de este tipo de agua (Fourqurean et al., 1993). Galvez et al. (1993) reportaron un embalse de cuenca cárstica y aguas duras donde el $\mathrm{P}$ no precipitó con el $\mathrm{CaCO}_{3}$. A pesar de las elevadas concentraciones de $\mathrm{Ca}$ presentes en las aguas de este embalse, el fósforo registró valores altos que clasificaron a las aguas como eutróficas, características que también se manifestaron en los altos valores de clorofila $a$ detectados.

Un estudio realizado por Morris \& Lewis (1988), reveló que la relación entre el nitrógeno inorgánico disuelto y el $\mathrm{P}_{T}$ era el mejor índice para explicar el nutriente limitante del desarrollo fitoplanctónico. Estos autores propusieron que las aguas interiores con valores de la relación inferiores a $0.5 \mathrm{mg} / \mathrm{L}$ estaban limitadas por 
nitrógeno, entre 0.5 y $4 \mathrm{mg} / \mathrm{L}$ por ambos nutrientes, y superiores a $4 \mathrm{mg} / \mathrm{L}$ limitadas por fósforo.

A pesar que el desarrollo fitoplanctónico en este embalse estuvo limitado por el nitrógeno, pudo haber ocurrido la incorporación del mismo desde el aire. Según Wetzel (1975), el nitrógeno atmosférico puede ser fijado por las cianobacterias, proceso que pudiera ocurrir en este ecosistema, considerando las altas concentraciones de este tipo de algas encontradas por Comas (2009) en el mismo momento que se realizó esta investigación.

Como el nutriente limitante del desarrollo fitoplanctónico fue el nitrógeno se infiere que para disminuir la eutrofización de las aguas se deben controlar los vertidos de este nutriente.

\section{CONCLUSIONES}

El agua del embalse Abreus resultó medianamente salina y de dureza elevada, con un marcado componente estacional que puede ser cuantificado mediante el cálculo de sus índices estacionales. Los componentes mayoritarios estuvieron altamente correlacionados, por lo que fue posible la reducción de variables mediante la aplicación del estudio de componentes principales.

El nuevo factor extraído denominado "mineralización" registró diferencia significativa entre los dos períodos evaluados (Noviembre-Mayo y Junio-Octubre) y explicó la estacionalidad de los componentes mayoritarios estudiados.

El comportamiento y la concentración de ambos nutrientes analizados fueron similares en ambos puntos de muestreo. Los valores de clorofila $a, \mathrm{P}_{T}$ y $\mathrm{P}-\mathrm{PO}_{4}$ permitieron clasificar el embalse como eutrófico y a pesar de los altos valores de $\mathrm{D}_{T}$, el $\mathrm{P}_{T}$ registró valores elevados.

El nitrógeno limitó el desarrollo fitoplanctónico en el embalse y por tanto constituye el elemento a controlar para mitigar la eutrofización de sus aguas.

\section{BIBLIOGRAFÍA}

[APHA] AMERICAN PUBLIC HEALTH ASSOCIATION. 1998. Standard methods for the examination of water and wastewater, $20^{\text {th }} \mathrm{ed}$. Washing- ton, DC. $648 \mathrm{pp}$.

ATOBATELE, O. E. \& O. A. UGWUMBA. 2008. Seasonal variation in the physicochemistry of a small tropical reservoir (Aiba Reservoir, Iwo, Osun, Nigeria). African Journal of Biotechnology, 7: 1962-1971.

BALISTRIERI, L. S., R. N. TEMPEL, L. L. STILLINGS \& L. A. SHEVENELL. 2006. Modeling spatial and temporal variations in temperature and salinity during stratification and overturn in Dexter Pit Lake, Tuscarora, Nevada, USA. Applied Geochemistry, 21: 1184-1203.

BETANCOURT, C., R. SUÁREZ \& L. TOLEDO. 2009. Ciclo anual del nitrógeno y el fósforo en el embalse Paso Bonito, Cienfuegos, Cuba. Limnetica, 28: 79-90.

BOUZA, R., M. TERNERO \& A. J. FERNÁNDEZ. 2008. Trend study and assessment of surface water quality in the Ebro River (Spain). Journal of $\mathrm{Hi}$ drology, 361: 227-239.

CARLSON, R. E. 1977. A trophic state index for lakes. Limnol.Oceanogr., 23: 361-369.

CHEN, Y. J. \& S. C. WU. 2007. Geochemistry of sediment phosphorus impacted by high calcium export from the catchments: A case Study. Environmental Engineering Science, 24(4): 550-562.

COMAS, A., A. MOREIRA, A. LEON \& S. URIZA. 2004. Apuntes para un diagnóstico del río Damují, Cienfuegos. Informe científico-técnico. Proyecto Territorial CITMA. 26 pp.

COMAS, A. 2009. Florecimiento de cianoprocariotas en los embalses Damují y Avilés. Informe técnico presentado al Consejo Científico del Centro de Estudios Ambientales de Cienfuegos. Cuba. 3 pp.

COPETTI, D. G. TARTARI, G. MORABITO, A. OGGIONI, E. LEGNANI \& J. IMBERGER. 2006. A biogeochemical model of Lake Pusiano (North Italy) and its use in the predictability of phytoplankton blooms: first preliminary results. J. Limnol., 65(1): 59-64.

DE LEÓN, L. \& G. CHALAR. 2003. Abundancia y diversidad del fitoplancton en el Embalse de Salto Grande (Argentina-Uruguay). Ciclo estacional y distribución espacial. Limnetica, 22(1-2): 103-113.

DIRNBERGER, J. M. \& J. WEINBERGER. 2005. Influences of Lake Level Changes on Reservoir Water Clarity in Allatoona Lake, Georgia. Lake Reserv. Manage., 21(1): 24-29.

DURFOR, C. N. \& E. BECKER. 1964. Public water supplies of the 100 largest cities in the United States, 1962. U.S. Geological Survey Water-Supply Paper 1812. 364 pp. 
ESTRADA, M. 1978. Relationship among biological and physicochemical parameters in Spanish reservoirs. Verh. int. Ver. Limnol., 20: 1642-1646.

FOURQUREAN, J. W., R. D. JONES \& J. C. ZIEMAN. 1993. Processes influencing water column nutrient characteristics and phosphorus limitation of phytoplankton biomass in Florida Bay, FL, USA: inferences from spatial distributions. Estuarine, Coastal and Shelf Science, 36: 295-314.

FOY, R. H. 1992. A phosphorus loading model for Northern Irish Lakes. Water Res., 26: 633-638.

GALVEZ, J. A., F. X. NIELL \& J. LUCENA. 1993. $\mathrm{C}: \mathrm{N}: \mathrm{P}$ ratio of settling seston in a eutrophic reservoir. Verhandlungen Internationale Vereinigung Limnologie, 24: 1390-1395.

GARCÍA, R., G. PARRA \& F. GUERRERO. 2001. Sedimentation of phosphorus fractions and temporal variation in the $\mathrm{C}: \mathrm{P}$ ratio in La Concepcion reservoir, southern Spain. New Zealand Journal of Marine and Freshwater Research, 35: 711-723.

GIBBS, R. J. 1970. Mechanisms controlling world water chemistry. Science, 170: 1088-1090.

KIM, Y. \& B. KIM. 2006. Application of a 2-Dimensional Water Quality Model (CE-QUAL-W2) to the Turbidity Interflow in a Deep Reservoir (Lake Soyang, Korea). Lake Reserv. Manage., 22(3): 213-222.

KIM, K., Y. NISHIMURA \& T. NAGATA. 2006. Role of dissolved organic matter in hypolimnetic mineralization of carbon and nitrogen in a large, monomictic lake. Limnol. Oceanogr., 51(1): 70-78.

LAIZ, O.\& E. FLORES. 2007. Análisis QuímicoGeológico de la Cuenca Hidrográfica del embalse Abreus. Informe científico técnico. Empresa de Investigaciones y Proyectos Hidráulicos Habana, Cuba. 26 pp.

LANGLET, D, L. Y. ALLEMAN, P. D. PLISNIER, H. HUGHES \& L. ANDRÉ. 2007. Manganese content records seasonal upwelling in Lake Tanganyika Mussels. Biogeosciences, 4: 195-203.

LIOU, M. L., S. L. LO \& C. Y. HU. 2003. Application of two-stage fuzzy set theory to river quality evaluation in Taiwan. Water Res., 37: 1406-1416.

LÓPEZ, P. \& J. A. MORGUÍ. 1993. Factors influencing fractional phosphorus composition in sediments of Spanish reservoirs. Hydrobiologia, 253: 73-82.

MARGALEF, R. 1983. Limnología, Ediciones Omega S.A., Barcelona, 1010 pp.

MEDINA, I. \& J. A. HERRERA. 2003. Spatial characterization of water quality in a karstic coastal lagoon without anthropogenic disturbance: a multivariate approach. Estuarine, Coastal and Shelf Science, 58: 455-465.

MORRIS, D. P. \& W. M. LEWIS. 1988. Phytoplanckton nutrient limitation in Colorado lakes. Freshwater Biol., 20: 315-327.

MWAURA, F. 2006. Some aspects of water quality characteristics in small shallow tropical man-made reservoirs in Kenya. African Journal of Science and Technology (AJST). Science and Engineering Series, 7(1): 82-96.

OECD. 1982. Eutrophication of Waters. Monitoring, Assessment and Control. Organisation for Economic Co-operation and Development, Paris, 154 pp.

RAKOCEVIC, J. \& H. HOLLERT. 2005. Phytoplankton Community and Chlorophyll $a$ as Trophic State Indices of Lake Skadar (Montenegro, Balkan). Environ Sci \& Pollut Res., 12(3): 146-152.

SERAFIM, A., M. MORAIS, P. GUILHERME, P. SARMENTO, M. RUIVO \& A. MAGRIC. 2006. Spatial and temporal heterogeneity in the Alqueva reservoir, Guadiana river, Portugal. Limnetica, 25(3): 771-786.

SERRUYA, C. \& U. POLLINGHER. 1983. Lakes of the warm belt. Cambridge University Press. Cambridge. 569 pp.

SHUMWAY, R. H. \& S. D. DTOFFER. 2006. Time Series Analysis and its Applications, $2^{\text {ond }}$ ed. Springer Verlag ed. 575 pp.

SIVADIER, F., J. M. THEBAULT, \& M. J. SALENCON. 1994. Total phosphorus budget in Pareloup reservoir. Hydroecol. Appl., 6: 115-138.

TAPIA, F. U., J. A. HERRERA \& M. L. AGUIRRE. 2008. Water quality variability and eutrophic trends in karstic tropical coastal lagoons of the Yucatán Peninsula. Estuarine, Coastal and Shelf Science, 76: 418-430.

TOLEDO, A. P., M. TALARICO, S. J. \& CHINEZ, E. G. AGUDO. 1983. A aplicação de modelos simplificados para a avaliação de processo da eutrofização em lagos e reservatórios tropicais. XIX Congresso Interamericano de Engenharia Sanitária e Ambiental. Camboriú, Brasil 57 pp.

STRASKRABA, M., I. DOSTÁLKOVÁ, J. HEJZLAR \& V. VYHNÁLEK. 1995. The effect of reservoirs on phosphorus concentration. Int. Revue ges. Hydrobiol., 80: 403-413.

WEI, W. S. 2005. Time Series Analysis: Univariate and Multivariate Methods, Addison-Wesley ed. 496 pp.

WETZEL, R. G. 1975. Limnology, W. B. Saunders Company, United States of América. 743 pp. 